\title{
Analysis of partial AZFc deletions in Malaysian infertile male subjects
}

\begin{abstract}
Complete deletions in the AZF ( $a, b$, and c) sub-regions of the Y-chromosome have been shown to contribute to unexplained male infertility. However, the role of partial AZFc deletions in male infertility remains to be verified. Three types of partial AZFc deletions have been identified. They are gr/gr, b1/b3, and b2/b3 deletions. A recent meta-analysis showed that ethnic and geographical factors might contribute to the association of partial AZFc deletions with male infertility. This study analyzed the association of partial AZFc deletions in Malaysian infertile males. Fifty two oligozoospermic infertile males and 63 fertile controls were recruited to this study. Screening for partial AZFc deletions was done using the two sequence-tagged sites approach (SY1291 and SY1191) which were analyzed using both the conventional PCR gel-electrophoresis and the high resolution melt, HRM method. Gr/gr deletions were found in $11.53 \%$ of the cases and $9.52 \%$ of the controls $(p=0.725)$. A B2/b3 deletion was found in one of the cases $(p=0.269)$. No B1/b3 deletions were identified in this study. The results of HRM analysis were consistent with those obtained using the conventional PCR gel-electrophoresis method. The HRM analysis was highly repeatable ( $95 \%$ limit of agreement was -0.0879 to 0.0871 for SY1191 melting temperature readings). In conclusion, our study showed that partial AZFc deletions were not associated with male infertility in Malaysian subjects. HRM analysis was a reliable, repeatable, fast, cost-effective, and semi-automated method which can be used for screening of partial AZFc deletions.
\end{abstract}

Keyword: AFZ; HRM; Male infertility; Partial deletions 